This item was submitted to Loughborough's Research Repository by the author.

Items in Figshare are protected by copyright, with all rights reserved, unless otherwise indicated.

\title{
A numerical study of the aspiration efficiency of a thin-walled sampler facing the wind for high velocity ratios
}

PLEASE CITE THE PUBLISHED VERSION

http://dx.doi.org/10.1016/j.jaerosci.2004.07.010

PUBLISHER

(C) Elsevier

VERSION

AM (Accepted Manuscript)

LICENCE

CC BY-NC-ND 4.0

\section{REPOSITORY RECORD}

Dunnett, Sarah J.. 2012. "A Numerical Study of the Aspiration Efficiency of a Thin-walled Sampler Facing the Wind for High Velocity Ratios". figshare. https://hdl.handle.net/2134/11256. 
This item was submitted to Loughborough's Institutional Repository (https://dspace.lboro.ac.uk/) by the author and is made available under the following Creative Commons Licence conditions.

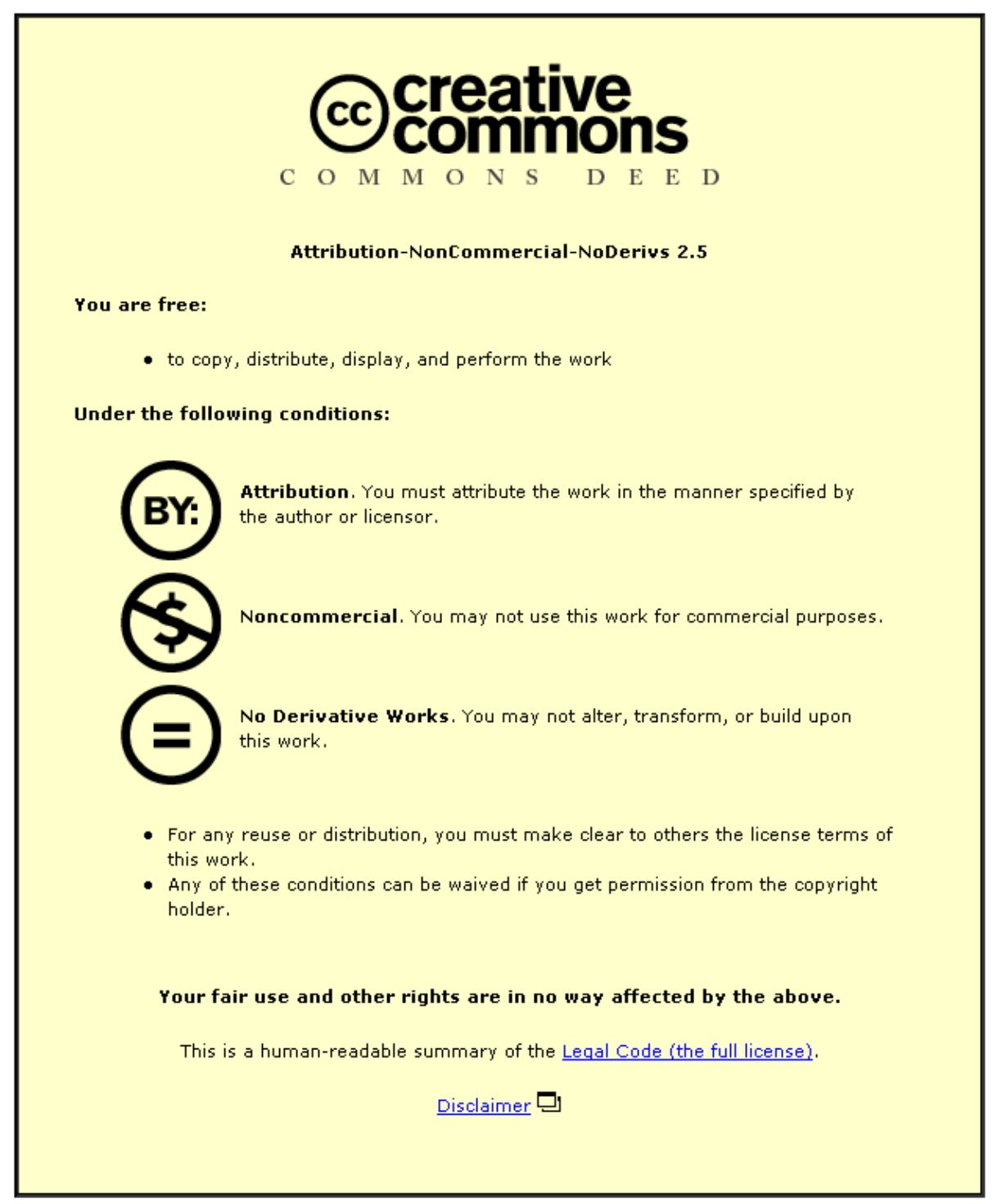

For the full text of this licence, please go to: http://creativecommons.org/licenses/by-nc-nd/2.5/ 
A numerical study of the aspiration efficiency of a thin-walled sampler facing the wind for high velocity ratios.

\author{
S.J.Dunnett \\ Department of Aeronautical and Automotive Engineering \\ Loughborough University \\ Loughborough LE11 3TU \\ U.K.
}

Running title: Sampling at high velocity ratios 


\begin{abstract}
In this work the fluid flow and particle trajectories for a thin-walled sampling probe facing the wind are considered. The flow around the sampler and into the sampler has been determined numerically and the paths of the particles in the flow are then traced and the efficiency of the sampler investigated.

A variety of operating conditions have been considered, in particular large values of the velocity ratio, $\mathrm{R}$, which is equal to the ratio of the undisturbed free stream air velocity to the average sampling velocity. The situation of large $\mathrm{R}$ values is becoming increasingly important as samplers are developed with low flow rates. Previous experimental results have shown that the empirical model developed for sampling in moving air does not accurately predict the efficiency of samplers operating at these high values of $\mathrm{R}$. The numerical results show that the aspiration efficiency for high $\mathrm{R}$ is not significantly affected by gravitational effects for the majority of cases of interest but it is dependent upon the magnitude of the reversal of the flow within the sampling tube.
\end{abstract}




\section{Introduction}

Aerosol samplers are widely used in health and environmental studies and hence have been a major area of research both theoretically and experimentally. Such samplers generally operate by withdrawing particle laden air through one, or more, orifices in their surface. The particles are then either collected on a filter, which is situated within the sampler, or they pass through a sensing region. The air flow near the sampler is distorted by the presence of the sampler and by the action of withdrawing air. Due to the forces acting on the particles within the flow they will not necessarily follow the air motion. In the case when all external forces acting on the particle, such as gravity, are negligible then the difference between the motion of the particle and that of the air will depend on the size of the Stokes number,

$$
\mathrm{St}=\frac{\mathrm{d}^{2} \gamma \mathrm{U}_{0}}{18 \mu \mathrm{D}}
$$

where $d$ is the particle diameter, $\gamma$ the particle density, $U_{0}$ the freestream velocity, $\mu$ the air viscosity and D the sampler diameter. Due to this inability of the particles to follow the air motion, the sample collected by the aerosol sampler may not be a true representation of the particle concentration in the undisturbed environment. A measure in the distortion of the sample collected is given by the aspiration coefficient, $\mathrm{A}$, which is defined as the ratio of the concentration of the particles in the air entering the sampler to that in the undisturbed flow.

Past work into understanding the performances of aerosol samplers includes both experimental and theoretical studies and much of the early work has been summarised by Vincent (1989). The studies have considered both thin-walled sampling probes and 'blunt' samplers. Thin-walled probes are the most idealised form of aerosol samplers and 
when the air speed and the sampling speed are the same then they collect 'representative' samples. They are widely used to provide reference samples by which the performances of other more complicated samplers can be determined. Although thin-walled samplers are idealised samplers, gaining an understanding of how they perform provides insights into the performance of more complicated samplers. Hence this area of aerosol sampling has received much attention, see, for example, Belyaev and Levin (1974), Jayasekera and Davies (1980), Grinshpun et al. (1990). In these studies the aspiration characteristics of thin-walled samplers were investigated for various operating parameters. However much of the published experimental data and theory on thin-walled samplers has been limited to a relatively narrow range of the velocity ratio,

$$
\mathrm{R}=\frac{\mathrm{U}_{0}}{\mathrm{U}_{\mathrm{S}}}
$$

where $U_{S}$ is the sampling velocity. The range of values of $R$ considered was generally restricted to $0.2 \leq \mathrm{R} \leq 6$. More recently work has been undertaken into understanding the performance of thin-walled samplers for values of R up to 50, see Paik and Vincent (2002a).

In the work of Belyaev and Levin (1974) a model was developed for the aspiration efficiency, A, of a thin-walled sampler facing the wind which has been widely accepted. Using physical reasoning it had previously been argued, Badzioch (1959), that A took the form

$$
\mathrm{A}=1+\alpha(\mathrm{R}-1)
$$

where $\alpha$ is the efficiency by which particles, due to their inability to follow the distorted air motion near the sampler, 'impact' onto the sampler inlet. Belyaev and Levin (1974) proposed that $\alpha$ be given by 


$$
\alpha=1-\frac{1}{1+\mathrm{G} \times \mathrm{St}}
$$

and obtained an empirical form for the quantity G given by

$$
\mathrm{G}=2+\frac{0.62}{\mathrm{R}}
$$

This expression was based on experimental data for values of $\mathrm{R}$ and $\mathrm{St}$ in the ranges $0.17 \leq \mathrm{R} \leq 5.56$ and $0.18 \leq \mathrm{St} \leq 2.03$. When considering larger values of $\mathrm{R}$ they argued, from physical considerations, that A could take the form

$$
\mathrm{A}=\mathrm{ER}+\frac{2}{\mathrm{R}}
$$

where

$$
\mathrm{E}=\frac{1.68 \mathrm{St}+0.13}{1.68 \mathrm{St}+1.13}
$$

and $\mathrm{E}$ is the efficiency of the inertial impaction of particles into the sampler at $\mathrm{U}_{\mathrm{S}}=0$. In recent years, interest has been stirred in studying aerosol aspiration for a much wider range of values of $\mathrm{R}$ than previously considered. For example, evidence now suggests that wind speeds found in workplaces, where ventilation is not forced, are typically less than $1 \mathrm{~m} / \mathrm{s}$, and this results in lower values of R than previously considered. Also, there is a desire to use lighter sampling pumps for industrial and environmental hygiene applications, see Vincent et al. (1999), which results in lower suction velocities and hence higher values of $\mathrm{R}$.

Some work was performed into understanding the performances of samplers operating at low sampling velocities, hence high values of R, by Yoshida et al. (1980) when they considered, theoretically and experimentally, thin-walled sampling probes oriented vertically, facing the oncoming flow. By investigating the particle deposition inside the sampling probes they postulated that some of the particles which collided with the internal 
walls of the probe were returned to the main air flow by a reversal of the air flow. Their work was restricted to one size of particle and vertically oriented samplers.

In the work of Liu et al (1989) a numerical study was made of the performance of a tube sampler for values of $\mathrm{R}$ up to 10 . They determined equations to fit their numerical data for the aspiration, penetration and loss coefficients. Where the penetration coefficient is the ratio of the particle concentration at the tube exit to that in the undisturbed flow and hence does not include particles that impact the tubes inner walls. The loss coefficient is the difference between the aspiration and penetration coefficients.

Recently, Paik and Vincent (2002a, 2002b) have studied the aspiration efficiency of samplers, both thin-walled and blunt, oriented horizontally, facing the wind for values of $\mathrm{R}$ up to 50. In their work the experimental results for aspiration efficiency are tabulated hence making it possible to compare them with theoretical models such as the one developed here. In their first study, Paik and Vincent (2002a) considered thin-walled samplers and found that their experimental data increasingly differed from the model developed by Belyaev and Levin (1974) as R increased above about 6. Using this data, they improved the Belyaev and Levin model, by altering the empirical term G, to more accurately describe what happens over the wider range of conditions considered. The form that $\mathrm{G}$ was postulated to take is given by

$$
\mathrm{G}=2+\frac{0.62}{\mathrm{R}}-0.9 \mathrm{R}^{0.1}
$$

As noted by Paik and Vincent (2002a), for some of the conditions they considered, the settling speed of the particles would have been of the same order of magnitude as the particles horizontal component of velocity across the sampling inlet. It would therefore be likely that gravitational effects may well affect the aspiration efficiency. The model of Belyaev and Levin, equations (3)-(5), was based purely on inertial considerations. 
Although Paik and Vincent (2002a) attempted to modify the value of G, equation (5), to include gravitational effects upon the particle motion and hence involve the Froude number, Fr, which represents the ratio between inertial and gravitational forces, they could not find a form that agreed with their experimental results as well as equation (6). The performance of blunt samplers for values of $\mathrm{R}$ up to 25 was considered by Paik and Vincent (2002b) and, as in the thin-walled case, they found that previous models developed for the aspiration efficiency significantly overestimated the aspiration as the value of $\mathrm{R}$ increases.

In this paper a numerical model is developed to investigate the behaviour of particles in the vicinity of a thin-walled sampler operating over a wide range of values of $\mathrm{R}$. An understanding of the flow and particle behaviour for the simpler thin-walled case very close to the sampler entry provides important insights into more complex blunt samplers. Further the effects of gravity upon the aspiration efficiency of the sampler are investigated.

\section{Formulation}

In order to make an initial investigation into sampler performance at large values of R, and to compare the results obtained with the available experimental data, a thin-walled sampler has been considered. The sampler, which has a suction velocity $\mathrm{U}_{\mathrm{S}}$, is assumed to be facing the air stream which is moving with an undisturbed uniform velocity $U_{0}$. A numerical model has been developed to predict the air flow around and into the sampler. In the model the flow is assumed to be ideal flow and the viscous effects neglected. This greatly simplifies the equations of motion for the flow and significantly reduces the 
computation time and resources necessary to obtain results. This model has been described in Dunnett and Wen (2002) and therefore it is not reproduced in detail in this paper.

Previously it has been found that the ideal flow model is an adequate model of the airflow for many sampling situations but it is possible that this is not always the situation. For the situation of interest in this work, sampling for large values of the velocity ratio $\mathrm{R}$, the air velocity in the sampling tube is significantly less than the undisturbed air velocity, and the limiting streamsurface reaches stagnation on the inside wall of the sampler at a short distance from the entry to the sampler, see Fig. 1. This distance will increase as $\mathrm{R}$ increases. The limiting streamsurface is the surface which separates the sampled air from that which is not sampled. It is possible that ideal flow will not model this adequately and the viscous effects are significant. The results obtained by the model described here will however predict the trend in sampler performance as $\mathrm{R}$ increases. To obtain accurate predictions of aspiration efficiency it may be necessary to model the flow field including the viscous effects and this more complex model is currently under development. In order to model the flow reversal within the sampling tube some modifications have been made to the potential flow model described by Dunnett and Wen (2002). The velocity plane, the plane inside the sampler at which the air velocity is assumed to take a constant value of $\mathrm{U}_{\mathrm{S}}$, is taken a distance $\mathrm{x}_{\mathrm{in}}$ inside the sampling tube, see Fig. 1. Mathematically and computationally this introduces corners into the geometry considered and hence inaccuracies in the mathematical method adopted. Every effort has been taken to minimise these inaccuracies. Also, in order to solve the problem numerically, the walls of the sampler have been taken to have a finite thickness and hence $\mathrm{D}$ and $\mathrm{D}_{\mathrm{i}}$ are taken to be the outer and inner diameter of the sampling tube, respectively. In assuming ideal flow the assumption has been made that the Reynolds number of the flow, given by 


$$
\operatorname{Re}=\frac{\mathrm{DU}_{0}}{\mathrm{v}}
$$

where $v$ is the kinematic viscosity of the air, is large. For the majority of the region of interest this will be a reasonable assumption as the value of Re is usually in the range $\mathrm{O}\left(10^{2}\right)-\mathrm{O}\left(10^{3}\right)$. However, viscous forces will be significant near the outer surface of the sampler, but this region is small and will not have a significant affect upon the motion of the particles. Inside the tube viscous effects will be significant in the boundary layer adjacent to the inside sampler walls. The thickness of the boundary layer will be small near the sampler entry but increase with distance down the tube. For a long tube the boundary layer thickens and eventually envelopes the whole flow, the flow is then said to be 'established'. However, in the samplers modelled here their lengths are assumed to be small and hence the boundary layer remains thin and viscous effects negligible.

Once the velocity of the air has been obtained, it is possible to trace the paths of the particles within the flow. In performing this calculation it was assumed that the particles were sufficiently small to cause no disturbance to the flow and that all external forces, except gravity, were negligible. The particle trajectories were obtained numerically by solving the non-dimensional equations of motion of the particles. In the situation when the sampler is pointing vertically upwards these equations, are given by, see Dunnett and Ingham (1988) 


$$
\begin{aligned}
& \frac{d u}{d t}=\frac{U-u}{S t} \\
& \frac{d v}{d t}=\frac{V-v}{S t}-\frac{1}{F r} \\
& \frac{d w}{d t}=\frac{W-w}{S t} \\
& u=\frac{d x}{d t}, v=\frac{d y}{d t}, w=\frac{d z}{d t}
\end{aligned}
$$

where $(\mathrm{U}, \mathrm{V}, \mathrm{W})$ and $(\mathrm{u}, \mathrm{v}, \mathrm{w})$ are the air and particle velocities, respectively, in the $(\mathrm{x}, \mathrm{y}, \mathrm{z})$ directions. The non-dimensional Stokes number, St, and Froude number, Fr, are given by

$$
\mathrm{St}=\frac{\mathrm{d}^{2} \rho \mathrm{U}_{0}}{18 \mu \mathrm{D}}, \quad \mathrm{Fr}=\frac{\mathrm{U}_{0}^{2}}{\mathrm{gD}}
$$

where $\rho$ is the particle density and $\mu$ the air viscosity.

In tracing the particle paths, a finite-difference method was used, see Dunnett and Ingham (1988). The particles were started at a very large distance from the sampler, where the flow was assumed to be undisturbed by the physical presence of the sampler. It was assumed that the particles were then moving with the same velocity as the airflow, $\mathrm{U}_{0}$, plus their settling velocity, vs.

The particles were then traced and the limiting particle trajectories, i.e. those separating the particles which are sampled from those that are not, were determined. The surface formed by these limiting trajectories will enclose an area $\mathrm{a}_{0}$ on the plane perpendicular to the initial direction of particle motion, in the undisturbed flow. If the effects of gravity are neglected when tracing the particle paths then this area will be circular and lie on the plane $\mathrm{x}=\mathrm{x}_{0}$, where $\mathrm{x}_{0}$ is a value of $\mathrm{x}$ at a large distance from the sampler. However when 
the effects of gravity are included, $\mathrm{a}_{0}$ is no longer circular and will lie on a plane inclined at an angle $\alpha$ to the horizontal, where $\tan \alpha=\frac{\mathrm{U}_{0}}{\mathrm{v}_{\mathrm{S}}}=\frac{\mathrm{Fr}}{\mathrm{St}}$.

The efficiency with which the sampler aspirates particles can be measured by the aspiration efficiency, A. This may be defined as the ratio of the concentration of particles passing through the sampler entry to that in the undisturbed air far away from the sampler. Hence $\mathrm{A}$ is given by

$$
\mathrm{A}=\frac{\mathrm{a}_{0} \mathrm{v}_{\text {int }}}{\mathrm{Q}}=\frac{4 \mathrm{a}_{0} \sqrt{\mathrm{U}_{0}^{2}+\mathrm{v}_{\mathrm{s}}^{2}}}{\pi \mathrm{D}_{\mathrm{i}}^{2} \mathrm{U}_{\mathrm{s}}}=\frac{4 \mathrm{a}_{0} \mathrm{R} \sqrt{1+(\mathrm{St} / \mathrm{Fr})^{2}}}{\pi \mathrm{D}_{\mathrm{i}}^{2}}
$$

where $\mathrm{v}_{\text {int }}$ is the initial particle velocity and $\mathrm{Q}$ is volume of air sampled per unit time.

Results for the aspiration efficiency have been obtained for various situations and a comparison is made between the results obtained and the available experimental data. In the case of large values of the velocity ratio $\mathrm{R}$, the non-dimensional velocity across the face of the sampling tube is small compared with the non-dimensional settling velocity of the particles and hence it is possible that gravitational effects will contribute to A. In this paper, results have also been obtained by neglecting gravitational effects, the usual assumption, and a study is made of the effects of gravity upon aspiration. 


\section{Results}

Initially results were obtained in order to compare with the early work of Yoshida et al. (1980). In their work a theoretical and experimental investigation was made into the flow behaviour and sampling efficiency of thin-walled tubes, oriented vertically, operating at low sampling velocities. Experimentally they obtained results for one size of particle, $24 \mu \mathrm{m}$, and 4 values of the flow velocity, $\mathrm{U}_{0}$, namely $3,6,15$ and $24 \mathrm{~m} / \mathrm{s}$. This resulted in values of St, $\mathrm{R}$ and Fr in the ranges, $0.56 \leq \mathrm{St} \leq 4.48,1 \leq \mathrm{R} \leq 100$ and $\mathrm{Fr}>100$. In Fig. 2., A is shown as a function of $\mathrm{R}$ for $\mathrm{St}=0.56$ with the sampler pointing vertically upwards. In the figure the experimental data of Yoshida et al. (1980), the predictions from the empirical model of Belyaev and Levin (1974) and the predictions of the improved Belyaev and Levin model, given by Paik and Vincent (2002a), are shown as well as the present numerical results. As can be seen from the figure, the numerical results, and the results predicted by the Belyaev and Levin (1974) model, increasingly overestimate the value of A as R increases. Similar observations were made by Yoshida et al (1980) when comparing experimental and theoretical results. They observed experimentally that there were particles attached to the sampler tube at a certain distance from the tube inlet but there were none near the inlet. It was hypothesised that this was due to a return to the main flow of some of the particles which collided with the internal walls of the tube by a reversal of the air flow. Hence in order to investigate whether the reversed flow does affect aspiration, results have been obtained for A taking the limiting particle trajectories to be those that meet the internal sampler walls at flow stagnation. This makes the assumption that any particles that meet the sampler walls between the inlet and flow stagnation are not sampled but returned to the main flow by the reversed air flow. These are also shown in Figure 2 labelled 'new numerical results'. In this case the results lie closer to the experimental data than the original numerical results, though the difference 
between the two increases as $\mathrm{R}$ increases. The empirical formula of Paik and Vincent (2002a) fits the experimental data well for $\mathrm{R}<30$. In obtaining the numerical results it was found that, in this case, the gravitational effects were negligible. It appears from these results that a major factor affecting the aspiration efficiency when sampling at large values of $\mathrm{R}$ is the flow reversal inside the sampling tube. Such effects were not included in the empirical model of Belyaev and Levin (1974) and hence could account for the discrepancies.

Results have also been obtained in order to compare with the experimental data of Paik and Vincent (2002a). In their experiments, the value of $\mathrm{R}$ ranged from 0.5 to 50, St from about 0.05 to 3.7 and Fr took the two values of approximately 10.2 and 15.44 . In the results shown here, the case of Fr $=10.2$ has been considered. In Fig. 3 (a) the numerical and experimental results for $\mathrm{A}$ are shown as a function of $\mathrm{R}$ for $\mathrm{St}=0.351$, with the sampler oriented with its axis horizontal. As before, the numerical model significantly overestimates the value of A. However, taking the limiting particle trajectories to be those that meet the internal sampler walls at flow stagnation, labelled 'new numerical results' as before brings the numerical predictions of A close to the experimental data. The effect of gravity upon the results was investigated but the differences of including and not including gravity were found to be insignificant and hence are not shown. Other values of St were considered and the same behaviour was found to occur, i.e. taking the limiting particle trajectories to be those that just pass across the entry to the sampler leads to a significant overestimation in the value of A. However, taking the limiting particle trajectories to be those that meet the internal sampler walls at flow stagnation, brings the numerical results into line with the experimental data. As St increases, the difference between the two sets of numerical results decreases, this can be explained by the fact that as St increases the particles are less able to follow the flow and hence are more likely to 
travel down the sampling tube without meeting the sampler walls. This can be seen in Fig. 3(b) where results are shown for $\mathrm{St}=2.43$. In this figure numerical results, for the case when the limiting particle trajectories are taken to be those that meet the internal sampler walls at flow stagnation, are also shown when gravitational effects have been neglected in the model, as a difference is discernable in this case, though it is not great.

Due to the low flow velocities across the sampling inlet it has been felt, previously, that gravity may be affecting the sampling performance of the sampler hence leading to the discrepancies seen between the results given by the empirical models and the experimental data. From the work undertaken in this paper, it appears that the greatest factor affecting the aspiration efficiency is the airflow reversal inside the sampling tube and it is not the gravitational effects. An example of when the gravitational effects do have a significant affect upon the predicted value of $\mathrm{A}$ is seen in Fig. 4 where $\mathrm{A}$ is plotted as a function of the Froude Number, Fr, for $\mathrm{St}=2.43$ and $\mathrm{R}=50$. The Froude number is effectively the ratio between the inertial and gravitational forces on the particles. These values of St and R have been shown as they correspond to the largest particle size and the lowest sampling velocity taken by Paik and Vincent (2002a). As can be observed from the figure gravitational effects do not become significant until Fr becomes small. At Fr $\approx 10$, the value corresponding to the experimental conditions in Paik and Vincent (2002a), the difference in the value of A obtained by neglecting gravity effects is approximately $4 \%$. It is not until $\mathrm{Fr} \approx 6$ that the difference between the values of A predicted by neglecting, and not neglecting, gravitational effects becomes as great as $10 \%$. For these conditions, with a sampler of diameter $10 \mathrm{~mm}$, that corresponds to $\mathrm{U}_{0} \approx 0.767 \mathrm{~m} / \mathrm{s}, \mathrm{U}_{\mathrm{S}} \approx 0.01534$ and $\mathrm{d} \approx 100 \mu \mathrm{m}$. Any differences between the values of A predicted by neglecting, and not neglecting, gravitational effects in the numerical model greater than $10 \%$ correspond to sampling conditions such that $\mathrm{U}_{0}<0.767 \mathrm{~m} / \mathrm{s}, \mathrm{U}_{\mathrm{S}}<0.01534$ and $\mathrm{d}>100 \mu \mathrm{m}$. Hence it 
would appear that in order for gravitational effects to be significant, the values of $U_{0}$ and $\mathrm{U}_{\mathrm{S}}$ must be very small and the particle size very large.

From the results shown it can be seen that the numerical model is consistently predicting higher aspiration efficiencies than those seen experimentally for $\mathrm{R}>6$. As mentioned previously, due to the neglect of viscous effects there are limitations in the numerical model for predicting the airflow inside the sampling tube. As the air moves down the tube the boundary-layer on the inner surface of the tube, where the viscous effects of the flow are important, will grow in size and hence the assumption of ideal flow will become increasingly inaccurate. This could cause some inaccuracies in the results obtained. However the 'entry length' required to establish fully developed flow in a pipe, where viscous forces dominate, is always considerably larger than the values of $\mathrm{x}_{\text {in }}$ considered in this paper. For example, for laminar flow the entry length is estimated to be, see Massey (1976), $0.057 * \mathrm{Re}^{*} \mathrm{D}$ where $\mathrm{Re}$ is the Reynolds number of the flow, and hence for $\mathrm{Re}=$ 1000 this gives a length of 57D. Hence for the situations considered in this paper, and practically, it would not be expected that the boundary-layer inside the tube would have a significant affect upon the particle motion. Also the accuracy of the numerical method decreases as the length $\mathrm{x}_{\text {in }}$ increases, although every effort was made to keep these inaccuracies to a minimum. Due to these problems, the range of $\mathrm{x}_{\mathrm{in}}$ considered was $\mathrm{x}_{\text {in }}<1.5$, although in any experimental set-up it is likely that its value will be larger. This could account for some of the differences in the values of A predicted by the numerical model and the available experimental data.

Although the potential model suffers from these inaccuracies it does predict the trends in sampler performance. In order to accurately predict the aspiration efficiency for large values of $\mathrm{R}$ a viscous model would need to be adopted to model the airflow. Such a model is currently under development. 
From the results shown in this paper, it has been shown that in the case of sampling for large values of $\mathrm{R}$, for most sampling situations, the effects of gravity upon the aspiration efficiency is insignificant. This is the situation generally assumed when developing numerical and empirical models for predicting sampler performance. However, from the numerical model it appears that a significant factor affecting aspiration is the flow reversal within the sampling tube. The larger the value of $\mathrm{R}$ the bigger this area of flow reversal will be. The extent of the effect the flow reversal has upon aspiration efficiency will depend upon the nature of the particles, liquid particles, for example, are unlikely to be removed from the internal walls by the reversed flow and hence will be sampled. In that case the trends in aspiration predicted by the original numerical model would be expected to be displayed. In the case of dry particles, it is possible that they may rebound or be reentrained from the internal walls into the reverse air flow. Reentrainment is dependent upon whether the drag force exerted by the air flow upon the particle is greater than the friction force exerted by the surface. Both removal mechanisms are dependent upon many factors including particle size, particle material, nature of the surface and flow properties. In the experimental data shown here, that of Yoshida et al (1980) and Paik and Vincent (2002a), dry aerosol were used with diameters of at least $13 \mu \mathrm{m}$ and hence removal is possible. It appears therefore from the work shown here that the reason this experimental data differs from the established empirical models is likely to be due to the reentrainment of particle into the reverse flow. The physical reasoning leading to equations (3) and (4) is still valid when sampling at large values of $\mathrm{R}$ if the form of $\mathrm{G}$ is modified to take into account the loss of particles due to the flow reversal. The form for $\mathrm{G}$ postulated by Paik and Vincent (2002a), eqn (8), appears to model well the available experimental data, for dry aerosol, but more data is required in order to validate, or not, this expression for the aspiration efficiency of tube samplers operating at large $\mathrm{R}$ values. 


\section{Conclusions}

From the preliminary results presented in this paper, it appears that the case of large $\mathrm{R}$ for aerosol sampling, a situation that is becoming increasingly important, is a more complex situation than previously considered. It appears that the effects of gravity upon aspiration are, in general negligible, but the reversal of the flow within the sampling tube is leading to a removal of particles from within the tube. This flow reversal for $\mathrm{R}>>1$ has been noted previously, see Vincent (1989), but its effects upon aspiration have not been previously studied in detail and this appears to be one of the major factors affecting the aspiration efficiency.

The empirical formula of Belyeav and Levin (1974), developed for sampling tubes and frequently used in the field, models well the aspiration of particles across the sampling inlet. However, in the formula, no account is taken of the flow reversal within the sampling tube removing from the sampler particles which initially crossed the inlet of the sampler, and hence for large values of $\mathrm{R}$ it increasingly overestimates the value of $\mathrm{A}$. The work described in this paper shows that in the majority of cases of practical interest, gravitational effects upon aspiration are negligible and hence the empirical form of the expression for A developed by Belyaev and Levin (1974) is correct but the empirical factor $\mathrm{G}$ requires modification to take into account the flow reversal inside the sampling tube.

Further, it has been shown that the potential flow model adopted here to model the airflow near the sampler becomes increasingly inaccurate as the value of $\mathrm{R}$ increases. This method has been adopted for many sampling problems and has been found to accurately predict the performance of samplers, see Dunnett and Wen (2002), and it has the 
advantage of using less computational resources than other methods. However, it appears that it is necessary to adopt a viscous model when sampling at large values of $\mathrm{R}$.

In conclusion, when modelling the sampler performance at large values of $\mathrm{R}$, a viscous model must be adopted for the flow field and the empirical models that are presently in use must be adapted to account for the flow reversal. The modification made by Paik and Vincent (2002a) appears to accurately model the situation but more data is required in order to fully verify this. 


\section{Acknowledgement}

The author wishes to thank Professor Derek Ingham and Dr X Wen for their many helpful comments and advice. 


\section{References}

Badzioch, S. (1959) Collection of gas borne dust particles by means of an aspirated sampling nozzle. Brit. J.App.Phy. 10, 26-32

Belyaev, S.P and Levin, L.M. (1974) Techniques for collection of representative aerosol samples, J. Aerosol Sci. 5, 325-338.

Dunnett, S.J. and Ingham, D.B. (1988) The mathematics of blunt body sampling, Lecture notes in Engineering, Springer-Verlag, London.

Dunnett, S.J. and Wen, X. (2002) A numerical study of the sampling efficiency of a tube sampler operating in calm air facing both vertically upwards and downwards, J. Aerosol Sci., 33, 1653-1665

Grinshpun, S.A., Lipatov, G.N. and Sutugin, A.G. (1990) Sampling errors in cylindrical nozzles, Aerosol Sci. Technol. 12, 716-740.

Jayasekera, P.N. and Davies, C.N. (1980) Aspiration below wind velocity of aerosols with sharp edged nozzles facing the wind, J. Aerosol Sci. 11, 535-547

Liu, B.Y.H., Zhang, Z.Q. and Kuehn, T.H. (1989) A numerical study of inertial errors in anisokinetic sampling, J. Aerosol Sci. 20, 367-380.

Massey, B.S. (1976) Mechanics of Fluids, Van Nostrand Reinhold Company Ltd, London.

Paik, S. and Vincent, J.H. (2002a) Aspiration efficiency for thin-walled nozzles facing the wind and for high velocity ratios, J. Aerosol Sci. 33, 705-720.

Paik, S. and Vincent, J.H. (2002b) Aspiration efficiency of disc shaped blunt nozzles facing the wind, for coarse particles and for high velocity ratios, J. Aerosol Sci. 33, 15091524.

Vincent, J.H. (1989) Aerosol Sampling, John Wiley \& Sons, Chichester. 
Vincent, J.H., Ramachandran, G., Thomassen, Y. and Keeler, G.J. (1999) Application of recent advances in aerosol sampling science towards the development of improved sampling devices: the way ahead. J. of Environ. Monitoring, 1, 285-292. Yoshida, H., Ii, Y., Masuda, H. and Iinoyo, K. (1980) Measurement errors in the dust concentration at very low sampling velocities, Funtai Kogaku Kaishi, vol. 17, 123-130, (in Japanese), HSE Translation 9204. 


\section{Figure Captions}

Figure 1. Schematic diagram of the air flow near a sampling tube operating at high R.

Figure 2. Aspiration efficiency as a function of velocity ratio, $R=\frac{U_{0}}{U_{S}}$, for $S t=0.56$ with the sampler facing vertically upwards.

Figure 3. Aspiration efficiency as a function of velocity ratio, $\mathrm{R}=\frac{\mathrm{U}_{0}}{\mathrm{U}_{\mathrm{S}}}$, with the sampler oriented with the axis horizontal for a) $\mathrm{St}=0.351$ and $\mathrm{b}) \mathrm{St}=2.43$.

Figure 4. Aspiration efficiency as a function of Froude Number, Fr, with the sampler oriented with the axis horizontal for $\mathrm{St}=2.43$ 


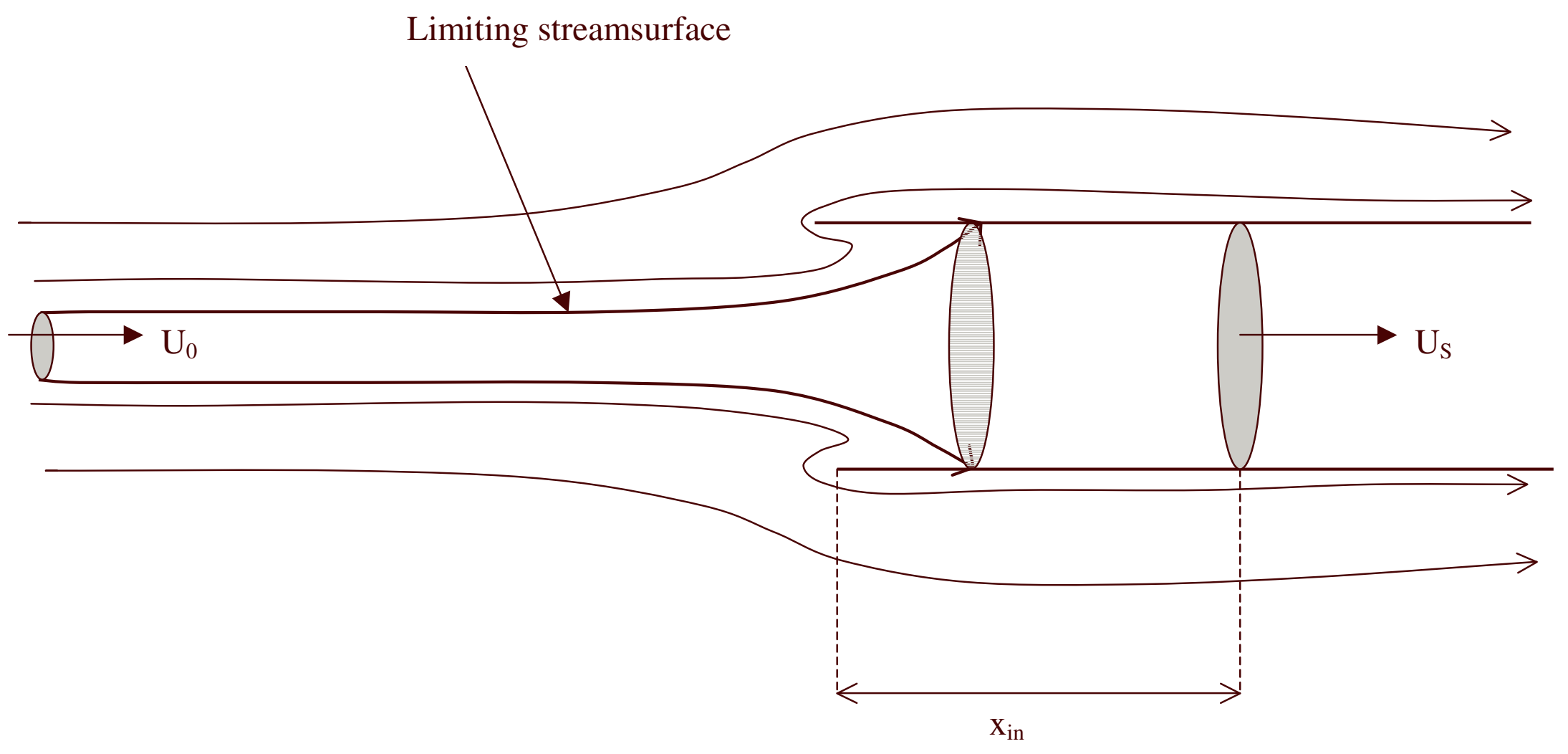

Figure 1 



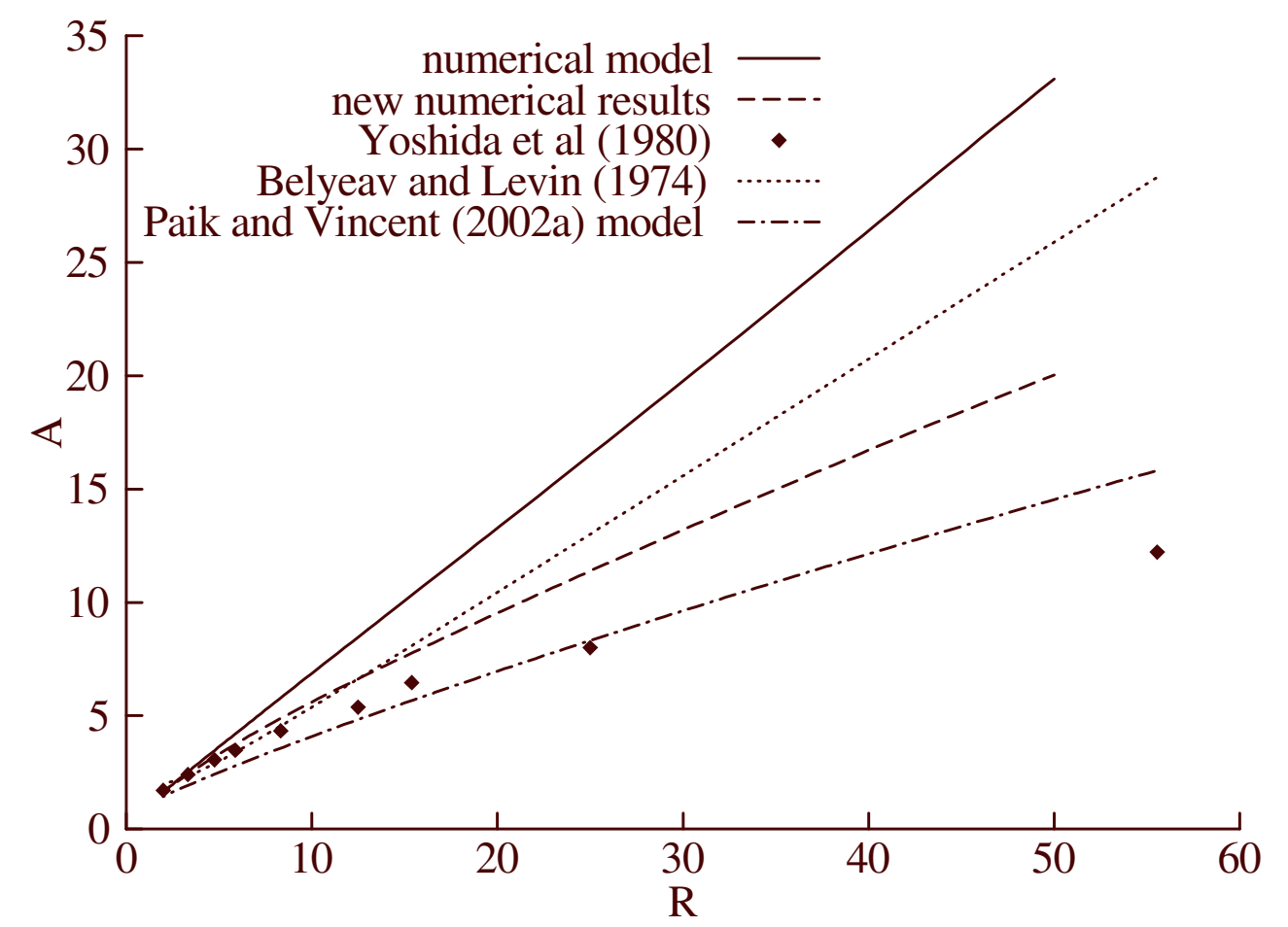

Figure 2

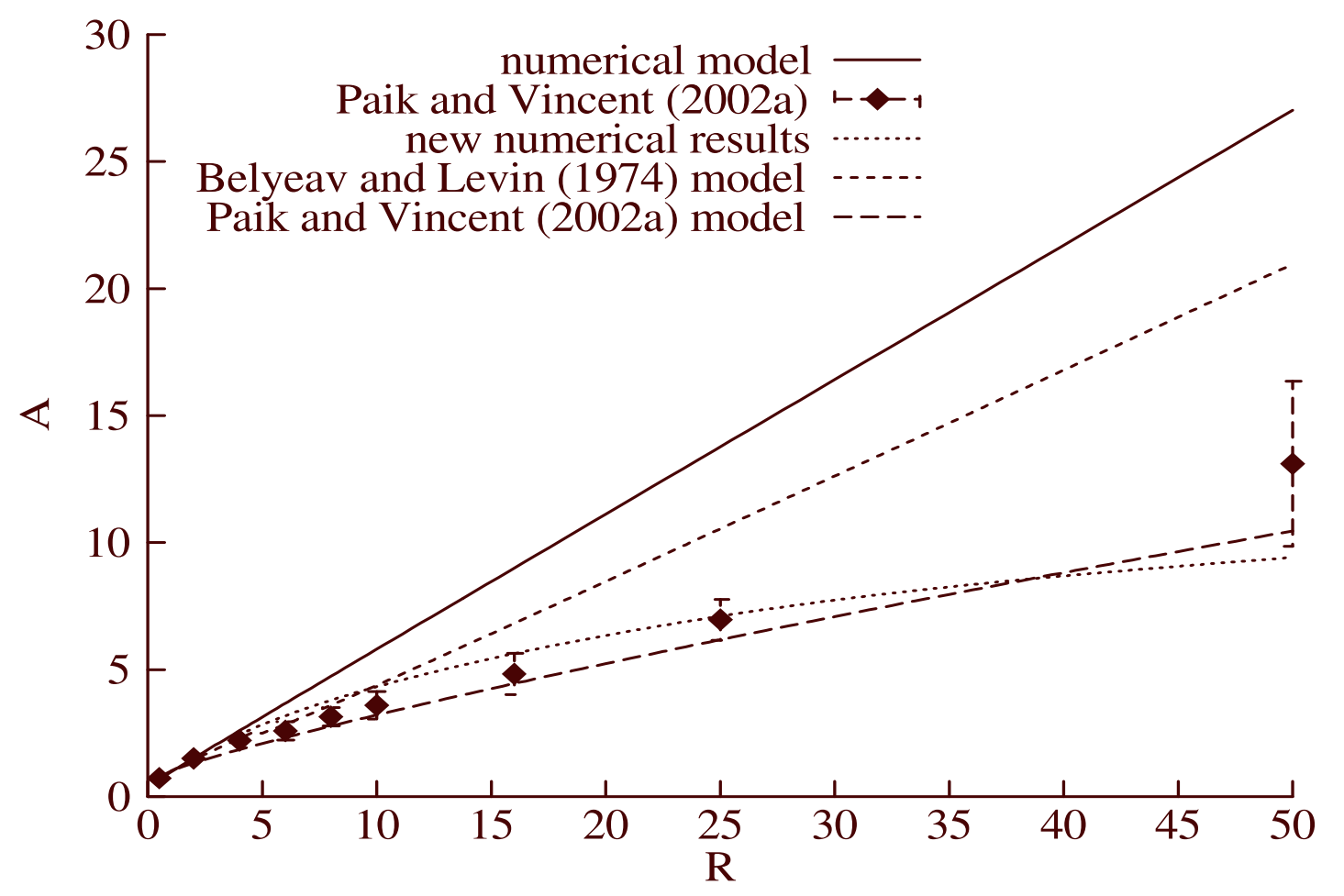

Figure 3(a) 


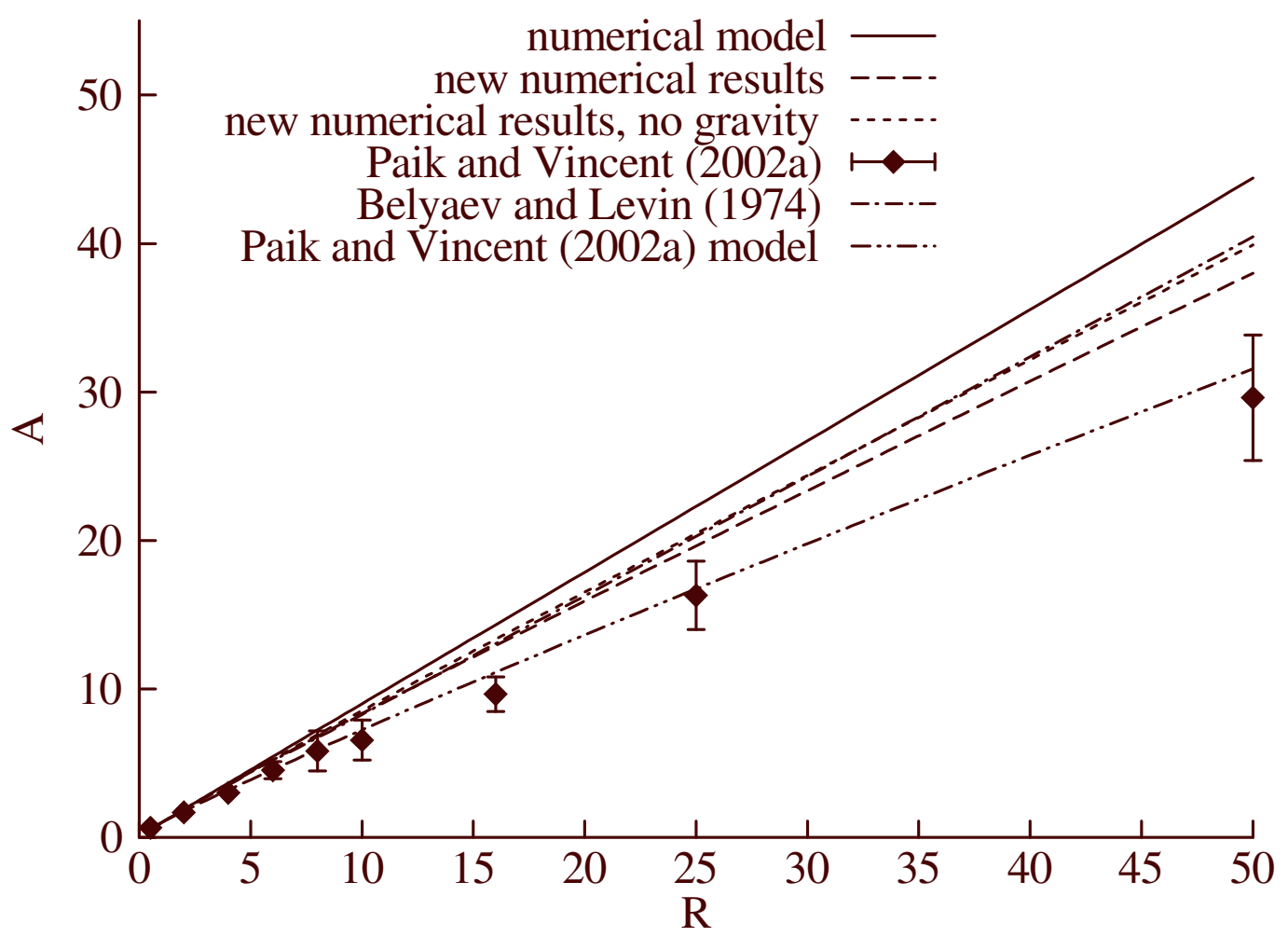

Figure 3(b)

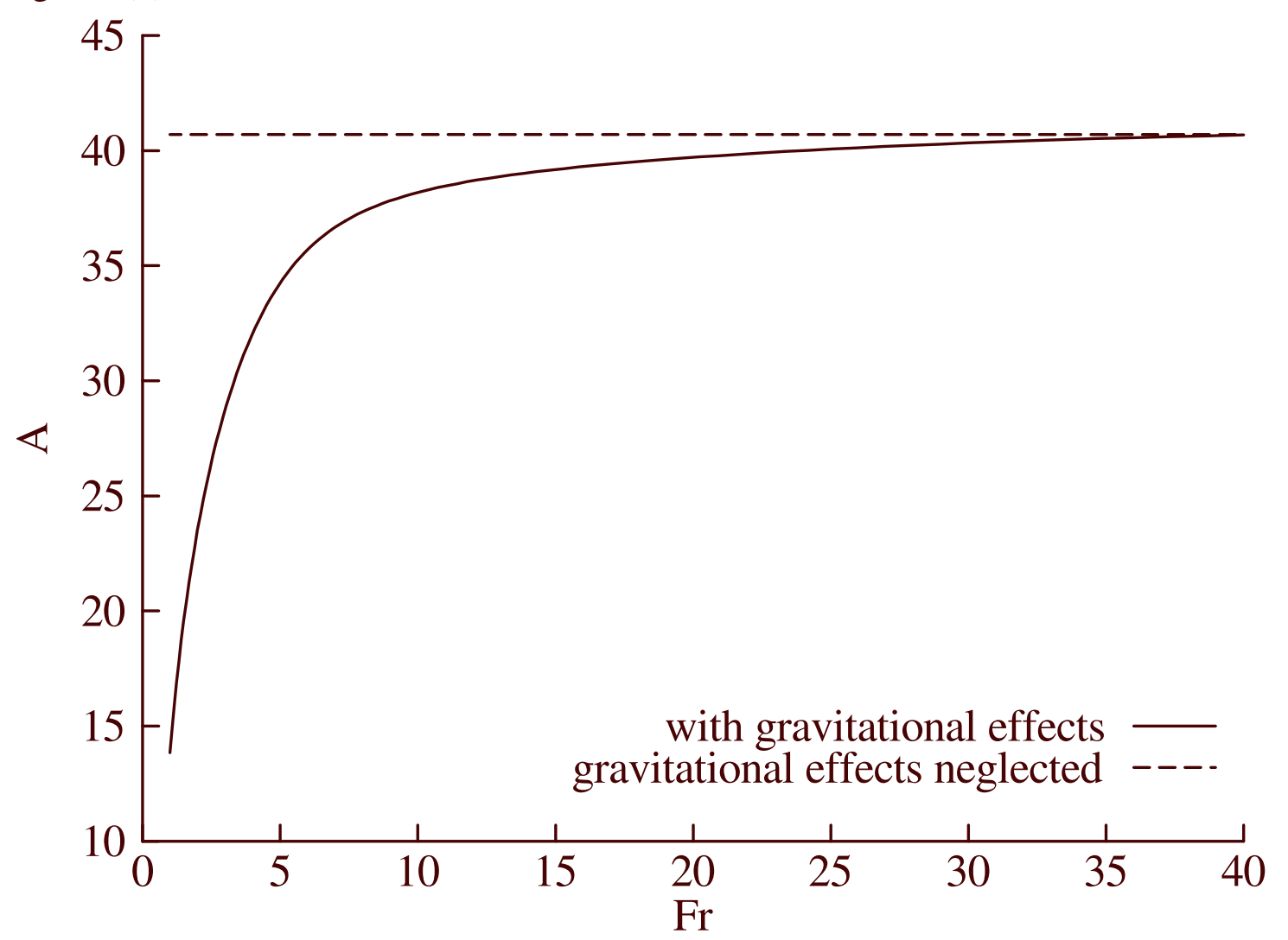

Figure 4 